\title{
Rendimiento de un motor de combustión interna utilizando diferentes mezclas carburantes.
}

\author{
Guillermo Becerra-Núñez ${ }^{\mathrm{a}, \mathrm{b}}$, Jorge Ovidio Aguilar Aguilar ${ }^{\mathrm{b}}$, Normand G. Chi Sanchez y Jesus \\ David Aviles Velazquez ${ }^{c}$.
}

(a) Consejo Nacional de Ciencia y Tecnología, Avenida Insurgentes Sur 1582, Crédito Constructor, Ciudad de México. C.P. 03940, México. (b) Universidad de Quintana Roo Boulevard bahía S/N, esq. Ignacio Comonfort, Col. del Bosque, Chetumal, Quintana Roo CP 77019, México, (c) Universidad Autónoma de Baja California Universidad 1, San Fernando, Tecate, Baja California, CP 21460, México. E-mail: catedra.guillermo@uqroo.edu.mx

\section{Recibido: 1 Junio 2021, aceptado: 20 de junio 2021}

\section{Resumen:}

En el presente trabajo se describen diferentes experimentos desarrollados con un motor de combustión interna, acondicionado principalmente como banco de pruebas. Inicialmente, el motor se utilizó como fuente de potencia para una motocicleta con gasolina, y se adaptó al banco de pruebas para analizar su desempeño con diferentes mezclas de combustibles. Las pruebas experimentales consideran el funcionamiento desde el reposo, manteniendo un régimen de velocidad gobernada como referencia, esto con el objetivo de registrar el consumo de combustible, la temperatura de operación para evitar el sobrecalentamiento y la composición de los gases de escape utilizando mezclas de combustible gasolina, con diésel y etanol al 5, 10 y $15 \%$ respectivamente. Los resultados obtenidos muestran funcionamientos similares del motor de combustión usando la mezcla de gasolina con diésel o etanol, alcanzando el mayor rendimiento de $18 \mathrm{seg} / \mathrm{ml}$, con la mezcla gasolina con diésel al 5\%. Finalmente, se observó que el motor de combustión no proporciona comportamientos adecuados con hidrógeno y biodiesel el utilizar el sistema de carburación implementado.

\section{Palabras Clave:}

Mezcla de combustibles, motor de combustión, rendimiento, gases de escape.

Fuel mixture, engine, performance, exhaust gases.

\section{Introducción}

Debido a la densidad energética de los combustibles líquidos, la facilidad de transportarlos, así como la diversidad de estaciones de recarga, los combustibles derivados del petróleo continúan siendo los más utilizados en México y en el mundo. Esto se puede observar fácilmente en los centros de abastecimiento cercanos a los lugares de trabajo, o bien indagando con las personas acerca del tipo de combustible que usan en sus medios de transporte. Adicionalmente, el mercado automotriz sigue utilizando el motor de combustión interna (MCI) como principal fuente de potencia de los vehículos terrestres. En [1] se proporcionó un análisis de ventas de vehículos con motor de combustión interna a nivel mundial en el año 2019, que representa más del $90 \%$ de los vehículos adquiridos en ese año. Sin embargo, los problemas de abastecimiento de combustibles y emisiones contaminantes están estrechamente ligados al sector autotransporte. La segunda problemática está asociada a la contingencia ambiental en ciudades principales, donde se establecen programas ambientales para regular las emisiones contaminantes, como el llamado "Hoy No Circula" de la Ciudad de México [2, 3], el cual consiste en reducir la movilidad de vehículos que utilizan MCI principalmente por usuarios particulares. Las razones anteriores motivan el desarrollo del presente trabajo con la finalidad de sustituir un porcentaje del combustible basado en petróleo, mediante bio-combustibles, considerando una fracción de masa o sustitución total como se describe en $[4,5,6]$, para proporcionar un adecuado funcionamiento del MCI o mejorar el desempeño con las mezclas adecuadas de hidrocarburos. Existen vehículos en el mercado que ya cuentan con tecnologías para el suministro de combustible flexible Flex Fuel, los cuales soportan los bio- 
combustibles. Aunque, la mayoría de los sistemas en vehículos convencionales no están preparados para sustituir una porción significativa del combustible original, lo cual forma parte de la motivación de los experimentos descritos en el presente trabajo.

Existen algunos trabajos enfocados al uso de biocombustibles con motores de combustión, en forma de gas, para generar energía eléctrica o bien para el uso vehicular, como se menciona en [7]. Además, algunos estudios con respecto a la mezcla de combustible fósil con biocombustible se describen en [8] usando gasolina con etanol para el motor de una motocicleta, adaptando el carburador para la inyección de etanol. En [9], se describe el comportamiento de un motor Kubota, similar al utilizado en el gasificador de All Power Labs [10,11], para el uso de diésel y mezclas con biodiesel. Asimismo, en [12] se estudió la mezcla etanol-diésel mediante las variaciones cíclicas de la combustión. En [13] se investiga una metodología de combustibles alternativos para motores diésel que se enfoca en etanol como agente aditivo en mezclas de biodiésel a partir de residuos de aceite de palma y de girasol. En [14], se estudió el etanol en un motor diésel, donde se inyecta el combustible diésel y se agrega un sistema auxiliar para el etanol, con lo que se consiguió una fracción máxima de masa de 0.634 de etanol.

En el presente trabajo, se analiza el comportamiento del motor de combustión para un sistema de carburación de motocicleta considerando la mezcla de gasolina con diésel o etanol, combinándolos con diferentes porcentajes de mezcla, y de la mezcla con hidrógeno y biodiésel, a diferencia de los combustibles mezclados en los trabajos previamente descritos. Los resultados experimentales muestran que el desempeño de un MCI es similar con las mezclas de gasolina con etanol o diésel, mientras que la combinación de hidrógeno y biodiesel no produce comportamientos adecuados con el sistema de carburación implementado.

El resto del trabajo se ordena de la siguiente forma; La sección 2 describe el desarrollo de los experimentos. Los resultados y su discusión se describen en la sección 3 y por último se mencionan algunas conclusiones y recomendaciones en la sección 4.

\section{Experimentos (Desarrollo)}

Para realizar los experimentos se acondicionó un banco de pruebas a partir de un motor de combustión interna que funcionaba con gasolina como combustible. El motor proviene de una motocicleta Suzuki mono cilíndrico, de 4 tiempos, encendido por chispa, enfriado por aire, con sistema de arranque eléctrico y por pedal. Se inició con la limpieza y ensamble de los componentes del motor, ya que tenía tiempo sin funcionar. En la Figura 1, se muestran los componentes que se limpiaron para el armado y puesta en servicio. Del lado derecho se aprecia la llanta trasera, al lado del cigüeñal, la camisa del pistón y la culata del motor. 


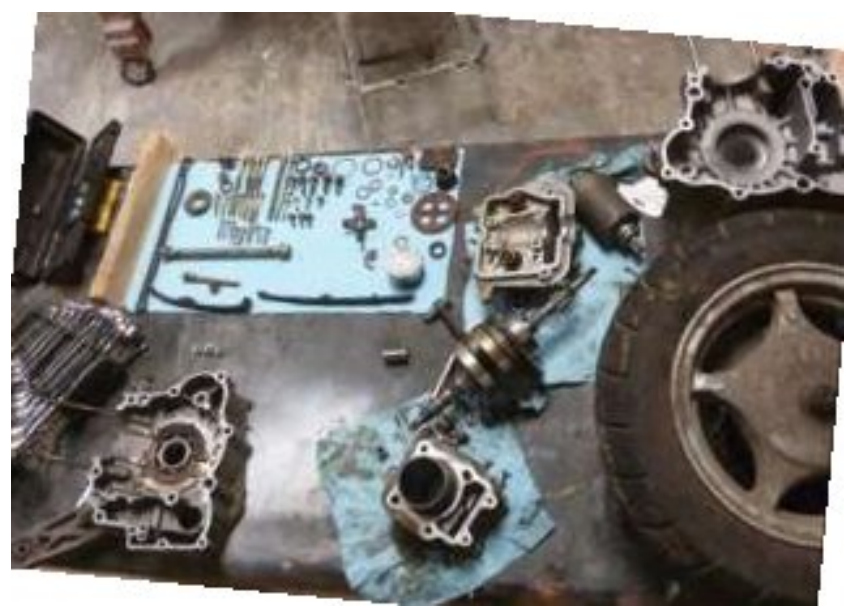

Figura 1. Elementos del motor de combustión para el armado y puesta en operación.

Durante el armado también se lograron medir los parámetros de carrera del pistón y diámetro del cilindro, con el propósito de determinar el volumen de la cámara de combustión (medida en centímetros cúbicos cc), además del volumen del múltiple de admisión, lo cual se describe en la tabla 1. En la figura 2 se muestran las mediciones físicas de los parámetros mencionados, además del armado completo del motor.

Tabla 1. Medición de parámetros

\begin{tabular}{|l|c|}
\hline \multicolumn{1}{|c|}{ Parámetro } & Valor \\
\hline Diámetro del cilindro & $51.70 \mathrm{~mm}$ \\
\hline Longitud de la carrera del pistón & $59.84 \mathrm{~mm}$ \\
\hline Volumen del múltiple de admisión & $60.00 \mathrm{~cm}^{3}$ \\
\hline
\end{tabular}

Cilindrada: $\frac{\pi}{4} D^{2} L=125,537.11 \mathrm{~mm}^{3}=125.537 c c$

(1)

Donde $D$ es el diámetro del cilindro o camisa en mm y $L$ es la carrera del pistón en mm

En el sistema del carburador, se implementó un arreglo de control con un microcontrolador Arduino, con la finalidad de establecer experimentos en régimen de velocidad gobernada, regulando la velocidad de salida por medio de un servomotor como actuador, que modifica la apertura de la válvula de aire (también conocida como mariposa) para acelerar y desacelerar el motor del sistema. 


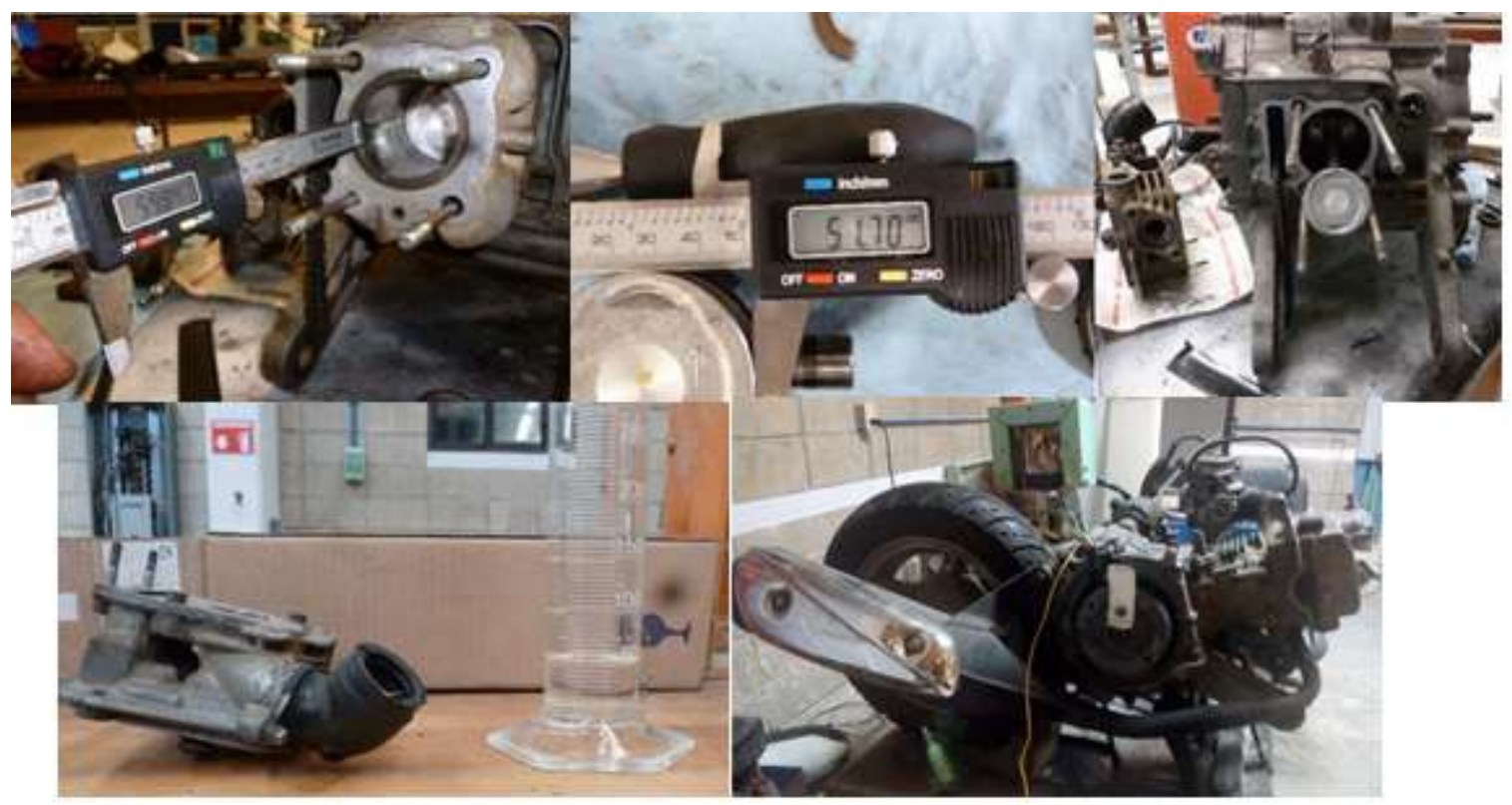

Figura 2. Medición de parámetros y armado del motor.

\section{Instrumentos de medición}

Por otro lado, se tienen diferentes instrumentos de medición necesarios para la comparación del desempeño del sistema. Primero, se utilizó una pipeta graduada y un cronómetro para medir el consumo de combustible, tomando en consideración el tiempo de demora en consumir 5 mililitros de combustible, y realizando este proceso en repetidas ocasiones para disminuir los errores de medición.

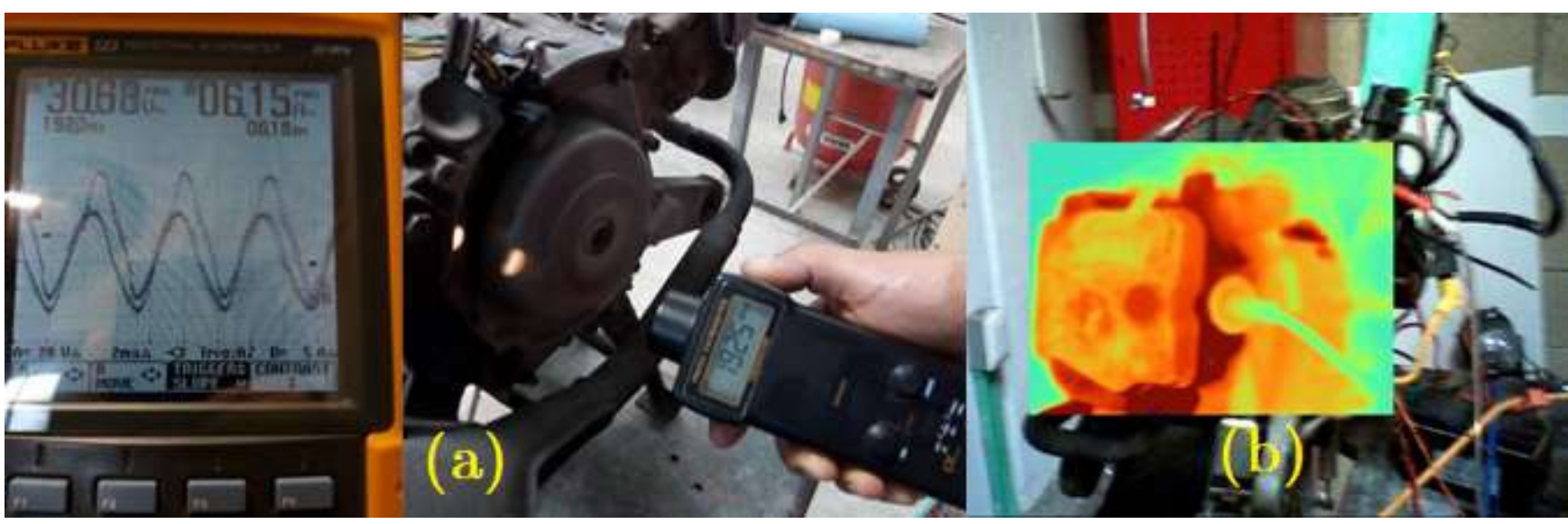

Figura 3. a) Medición de velocidad del MCI (tacómetro = 1,925 RPM) con relación a la frecuencia del generador $(192 \mathrm{~Hz})$, del lado izquierdo. b) Imagen del incremento de temperatura con la cámara termográfica. 
Para la medición de velocidad en el MCI, se tomó la señal de frecuencia, a partir del generador eléctrico de 12 polos que se acopla en el cigüeñal, ya que tal sistema indica la posición para la chispa de la bujía. Este sistema funciona cuando el motor está en operación. El imán que gira alrededor del generador también actúa como volante de inercia, el cual cuenta con un imán resaltado que al pasar cerca de un sensor de efecto hall, este envía un pulso a la bobina, y a su vez esta manda un alto voltaje a la bujía para realizar la explosión de la mezcla aire/combustible en la cámara de combustión. En la figura 3a, se confirma el valor de la frecuencia del generador de imán permanente correspondiente con la velocidad del motor de combustión, los cuales están relacionados por el número de polos, como se menciona en [15],

Velocidad $(\mathrm{RPM})=120 *($ Frecuencia $\mathrm{Hz}) /($ No. de polos $)$.

(2)

Para prevenir el sobrecalentamiento del motor, se monitorea la temperatura de operación usando un termopar tipo K, colocado entre la cabeza y la camisa del motor. La medición de temperatura se corroboró mediante una cámara termográfica Fluke modelo TiR1, cuya imagen presentada en la Figura $3 \mathrm{~b}$ representa el incremento la temperatura por el funcionamiento del MCI.

A partir del registro de la composición de los gases de escape, se determina que tan buena es la combustión y si la mezcla enriquecida con la que se alimenta la cámara de combustión es apropiada, de tal manera que se reduzca la emisión de gases de polución atmosférica. Para lo cual, se utilizó el instrumento analizador de gases de escape para el MCI (AGA5000 VEI) que se muestra en la figura 4, el cual, censa el porcentaje de gases en las emisiones del escape y así como las partículas de hidrocarburo no quemado. Los gases que se miden son: (i) Monóxido de carbono (CO), se produce cuando hay poco oxígeno disponible para la combustión y por tanto no llega para quemar todo el carbono del combustible completamente, quedando átomos de carbono unidos a solo un oxígeno formando el CO. (ii) Dióxido de carbono (CO2), que se produce al ser quemados los combustibles que contienen carbono. El carbono se combina durante esa operación con el oxígeno aspirado, es un gas incoloro, no combustible. (iii) óxidos de nitrógeno (N Ox), resulta al combinarse el oxígeno y el nitrógeno debido a las altas temperaturas que se alcanzan dentro del motor y a las altas presiones. En la cámara de combustión se forma el NO y al abrirse la válvula de escape los gases pasan al conducto de escape donde se combinan con oxígeno para formar NO2. (iv) Hidrocarburo no quemado (HC), son restos no quemados del combustible, que surgen en los gases de escape después de una combustión incompleta. La mala combustión puede ser debido a la falta de oxígeno durante la combustión (mezcla rica) o también por una baja velocidad de inflamación (mezcla pobre), por lo que es conveniente ajustar la riqueza de la mezcla. 


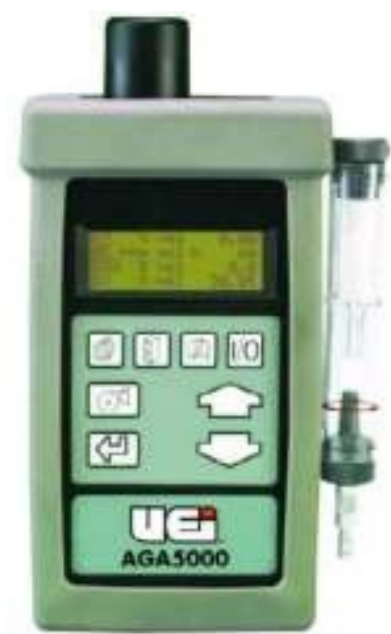

\section{Combustibles}

Figura 4. Analizador de gases de escape AGA5000.

A continuación, se describen los combustibles derivados del petróleo que fueron utilizados en los experimentos. Primero la gasolina por el funcionamiento inicial del motor, el cual se inyecta mediante el carburador, cuyo principio básico es aprovechar el vacío que deja el pistón y con el empuje de la presión atmosférica, se suministra la mezcla de aire y gasolina en el múltiple de admisión, para cuando se abre la válvula ingrese a la cámara de combustión. La cantidad de oxígeno ideal (O2), para que se lleve a cabo la explosión del combustible (CxHy) - hidrocarburo, está dada por la expresión de estructura molar.

$\mathrm{CxHy}+(\mathrm{x}+\mathrm{y} / 4)(\mathrm{O} 2)->\mathrm{xCO} 2+(\mathrm{y} / 2) \mathrm{H} 2 \mathrm{O}^{1}$

donde $\mathrm{C}$ es carbono, $\mathrm{H}$ hidrógeno y después de la combustión, se obtiene dióxido de carbono $\mathrm{CO} 2$ y agua $(\mathrm{H} 2 \mathrm{O})$.

De los hidrocarburos con que se experimenta, por un lado, la gasolina emplea el octanaje como la medida de resistencia a la explosión por compresión en el MCI. En términos generales, la característica principal para tener una gasolina adecuada es la que tiene mayor octanaje, lo cual, es el valor que muestra la resistencia del combustible a ser detonado prematuramente [18], después de que se comprima al interior de la cámara de combustión. Se refiere a la presión y temperatura a la que puede someterse la gasolina mezclada con aire antes de realizar la explosión. Por otro lado, para el diésel no se le puede nombrar octanos debido a que no necesita un punto de inflamación, la diferencia consiste en la elevada presión para su detonación, por lo que se le nombra cetano [19], ya que esté número es una medida de las características del encendido del combustible.

Por parte de los combustibles biológicos (biocombustibles) [20], se obtienen a partir de restos orgánicos, de los cuales se consideran de primera generación el bioetanol, el biodiésel y el biogás. Los cuales, se producen a partir de biomasa, la producción de etanol deriva de cultivos energéticos de maíz, sorgo, yuca, caña de azúcar, entre otros. El biodiésel se produce a partir de las oleaginosas de la soya, aceite de palma, colza, jatropha curca, etc. Por otro lado, el biogás se obtiene a partir de residuos orgánicos mediante procesos de digestión anaerobia de forma análoga al gas de síntesis, pero el último por el proceso de gasificación.

1 Se considera $\left(\mathrm{C}_{8} \mathrm{H}_{18}\right)$ para la gasolina y $\left(\mathrm{C}_{12} \mathrm{H}_{26}\right)$ para diésel, según [16] y [17]. 


\section{Mezclas de enriquecimiento de combustible.}

Para el enriquecimiento del combustible se hicieron varias mezclas de gasolina con distintos combustibles y biocombustibles empleados para los motores de combustión. Para lo cual, se etiquetan en tres porcentajes, por cantidad de volumen de combustible original a reemplazar en el MCI. Para la mezcla de gasolina con etanol se etiqueta con el prefijo "E\%" donde el símbolo \% representa el porcentaje de etanol que sustituye a la gasolina para realizar las pruebas. Es decir, para una mezcla de gasolina con 5\% de etanol la etiqueta es "E5", para el enriquecimiento del 15\% se etiqueta con "E15". Se mezclaron dos hidrocarburos y la etiqueta se designa con la inicial de ambos, seguido con el porcentaje de sustitución, como en los anteriores. Por ejemplo, para el caso de sustituir el 5\% de gasolina con diésel, se emplea la etiqueta "GD5", para el 10\% es "GD10". La mezcla de los mencionados combustibles/biocombustibles líquidos se realizó antes de ingresar al carburador.

\section{Resultados y discusión}

Los primeros experimentos se realizaron con el motor de combustión y el combustible original (gasolina), las mediciones consisten en analizar el consumo, la temperatura, velocidad y gases de escape a la salida, también se debe aclarar que el motor se operó con la misma carga del generador y transmisión acoplada, algunos resultados preliminares se presentan en [21]. Además, se aclara que el rendimiento de un automóvil o motocicleta está dado por el kilometraje recorrido por cada litro consumido, esto solo aplica en motores que sirven para la movilidad. Sin embargo, para los motores estacionarios (como en el presente trabajo), en su mayoría de generación eléctrica, el rendimiento se mide en horas de operación por litro consumido.

La figura 5 muestra las dinámicas de la velocidad del motor de combustión hasta llegar a estabilizarse alrededor de 2,450 RPM, en la misma figura también se observa como la temperatura llega a superar ligeramente los $85^{\circ} \mathrm{C}$, sin mayor incremento después de $1 \mathrm{~h}$ de operación.

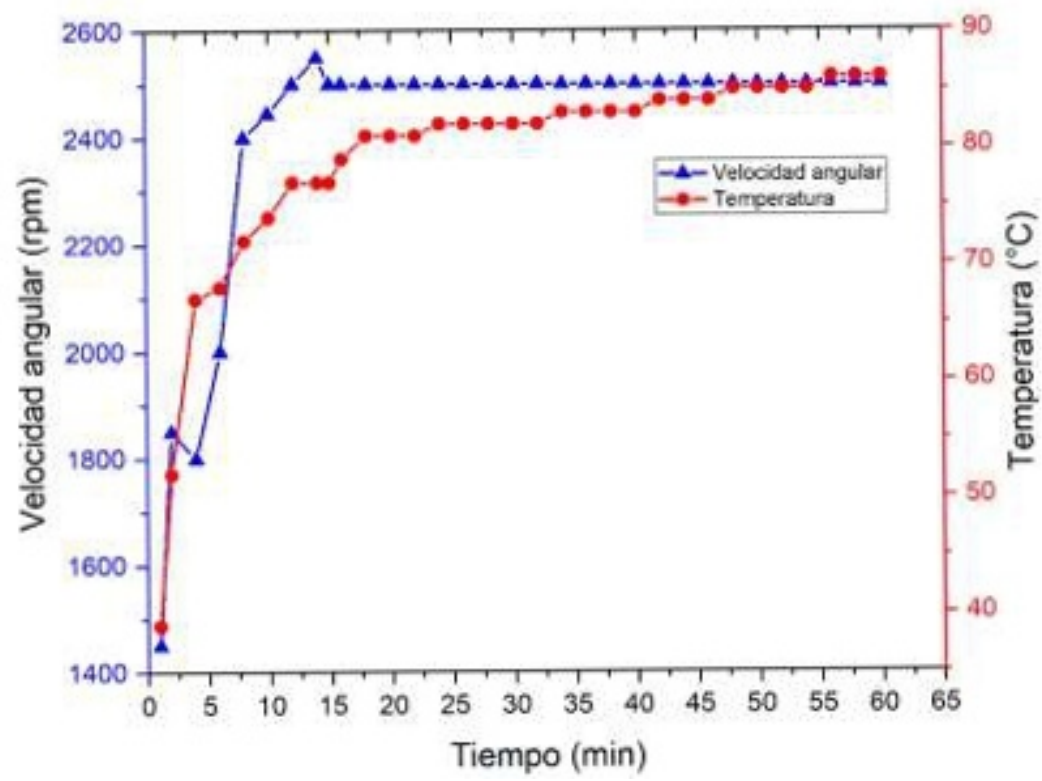


Figura 5. Comportamiento de la velocidad y temperatura del motor de combustión desde el arranque hasta que se estabiliza.

Por otro lado, en la figura 6 se muestra además el comportamiento dinámico de velocidad, el rendimiento del MCI medido en ( $\mathrm{seg} / \mathrm{ml})$, lo cual, es un parámetro principal para comparar el desempeño con la operación del motor con mezcla de combustibles.

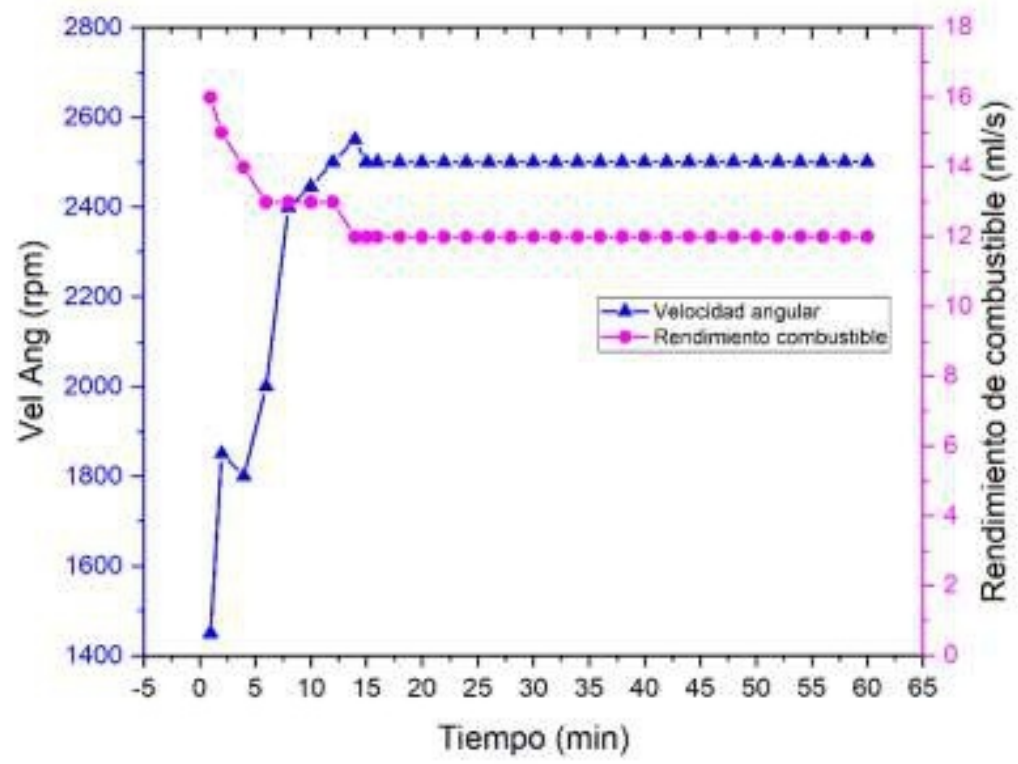

Figura 6. Dinámica del rendimiento y la velocidad del motor de combustión.

Los siguientes resultados se agrupan para analizar de mejor forma la comparación de la operación del motor con las mezclas gasolina con etanol y diésel, las etiquetas y proporciones se describen en la tabla 1 .

Tabla 2. Etiquetas de las mezclas de combustibles

\begin{tabular}{|c|c|c|c|}
\hline $\begin{array}{c}\text { Etiqueta } \\
\text { de mezcla }\end{array}$ & $\begin{array}{c}\text { Porcentaje } \\
\text { de gasolina }\end{array}$ & $\begin{array}{c}\text { Porcentaje } \\
\text { de etanol }\end{array}$ & $\begin{array}{c}\text { Porcentaje } \\
\text { de diésel }\end{array}$ \\
\hline Gasolina & $100 \%$ & $0 \%$ & $0 \%$ \\
\hline E5 & $95 \%$ & $5 \%$ & $0 \%$ \\
\hline E10 & $90 \%$ & $10 \%$ & $0 \%$ \\
\hline E15 & $85 \%$ & $15 \%$ & $0 \%$ \\
\hline GD5 & $95 \%$ & $0 \%$ & $5 \%$ \\
\hline GD10 & $90 \%$ & $0 \%$ & $10 \%$ \\
\hline GD15 & $85 \%$ & $0 \%$ & $15 \%$ \\
\hline
\end{tabular}

La figura 7 muestra las dinámicas de velocidad del motor en RPM, que para todos los casos se intentó mantener en un valor cercano a 2,450 RPM después de los primeros 10 minutos de operación, con el fin de comparar los otros desempeños del funcionamiento del MCI. 


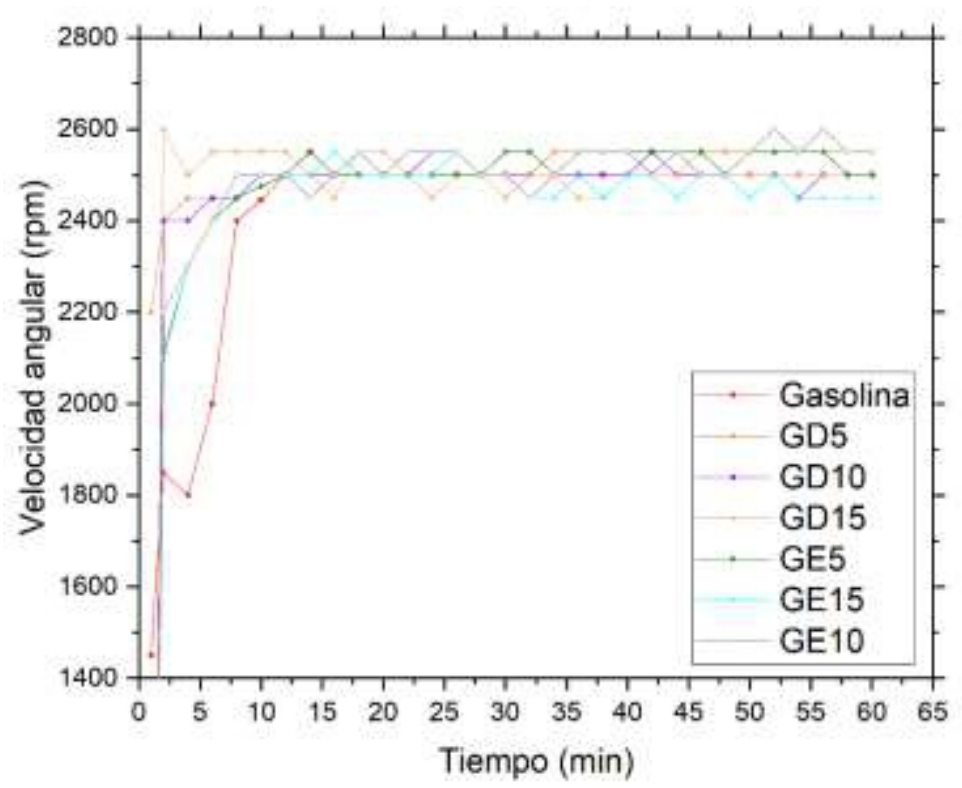

Figura 7. Dinámicas de la velocidad en (RPM) del motor de combustión para gasolina y mezcla con etanol al 5\%, 10\% y 15\%, así como la mezcla gasolina con diésel al 5\%, 10\% y 15\%.

En la figura 8, se muestran los perfiles de temperatura, tanto para gasolina como para las mezclas con etanol y diésel, cuando el motor funciona solo con gasolina presenta la menor temperatura, ligeramente mayor a $85{ }^{\circ} \mathrm{C}$ y cuando funciona con la mezcla de gasolina y $10 \%$ de etanol (etiqueta E10) tiene un valor cercano a $89^{\circ} \mathrm{C}$. Las demás dinámicas de temperatura se encuentran entre este rango de valores, para los enriquecimientos de E5, E15, GD5, GD10 y GD15.

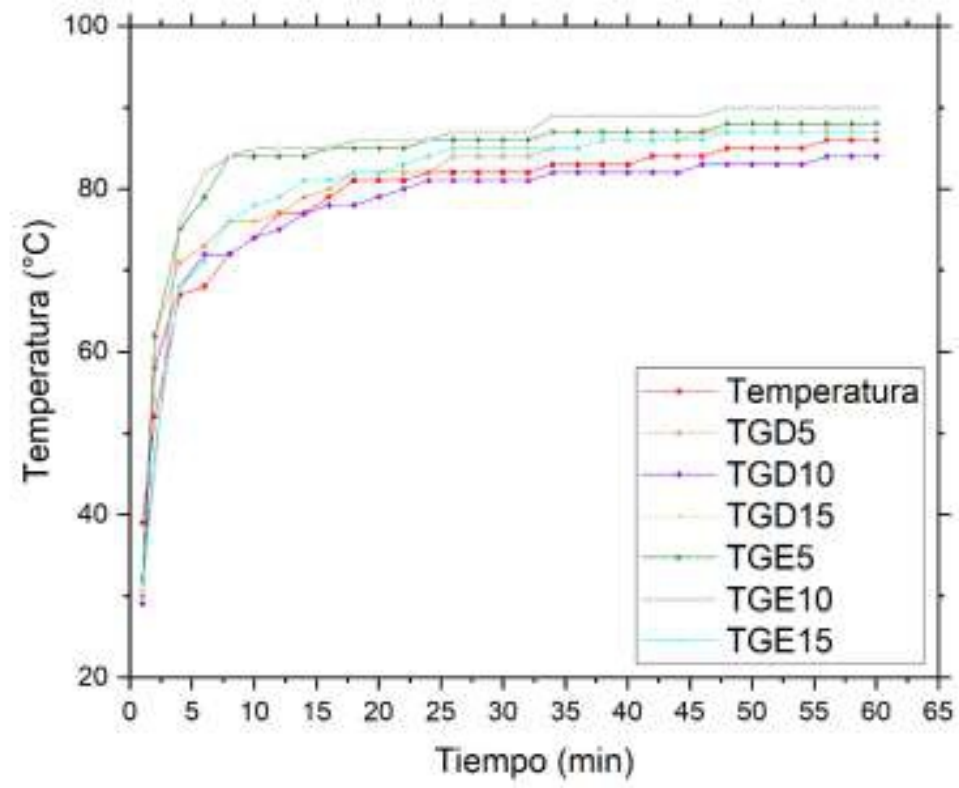

Figura 8. Dinámicas de la temperatura del motor de combustión para gasolina y mezcla con etanol E al 5\%, $10 \%$ y $15 \%$, así como la mezcla con diésel GD al 5\%, 10\% y $15 \%$.

Los resultados de rendimiento se muestran en la figura 9, donde el mayor rendimiento se presenta con la mezcla de gasolina con diésel al 5\% GD5, que después de 15 minutos se mantiene en un 
valor cercano a los $18 \mathrm{seg} / \mathrm{ml}$, después le sigue el rendimiento de la mezcla gasolina con etanol al $10 \%$ E10, que se sostienen en $16 \mathrm{seg} / \mathrm{ml}$, y el resto de las otras mezclas se encuentran por debajo de estos valores, como se puede apreciar en la figura.

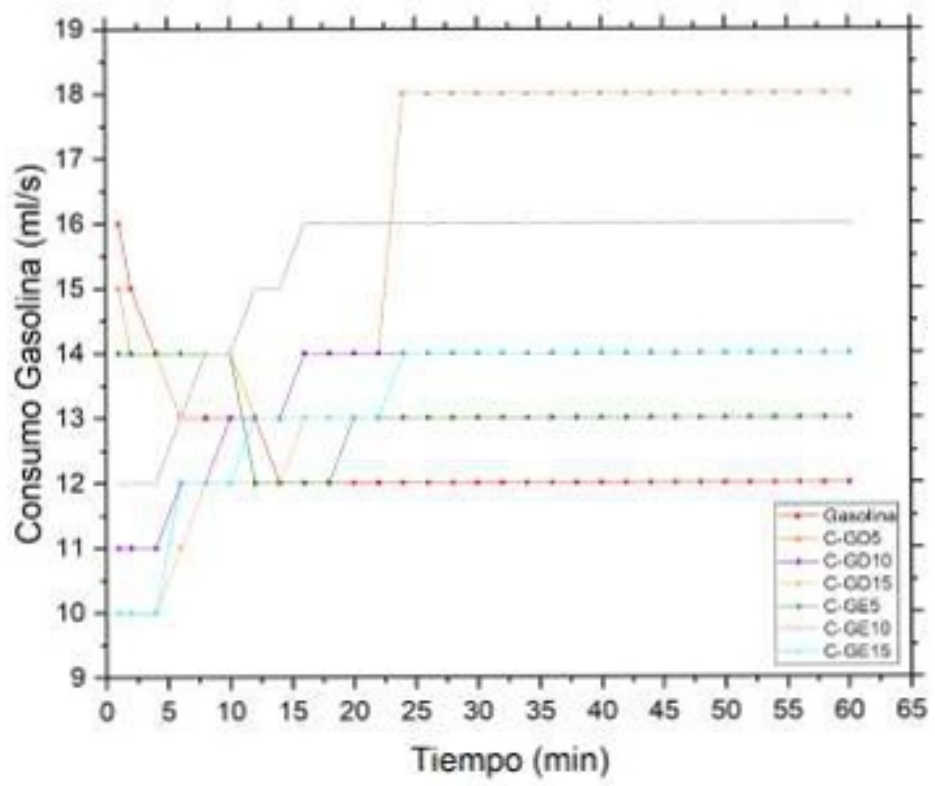

Figura 9. Dinámicas del rendimiento del motor de combustión para gasolina y mezcla con etanol E5 al 5\%, E10 - 10\% y E15 - 15\%, así como la mezcla con diésel GD al 5\%, 10\% y 15\%, respectivamente.

La tabla 2 muestra las lecturas de las mediciones de los gases de escape, las cuales se obtienen en los últimos 15 minutos de la prueba, donde se estabiliza el motor y prácticamente no hay variación en los valores. Se observa que las mezclas de gasolina con etanol y diésel se mantienen por debajo del valor obtenido cuando el motor opera solo con gasolina, así como el resto de las mediciones con la excepción de la mezcla gasolina con diésel al $15 \%$ para $\mathrm{HC}$ y NOx.

Tabla 3. Lecturas de gases de escape con gasolina y mezclas.

\begin{tabular}{|l|c|c|c|c|c|c|c|c|}
\hline Lectura & Limite & $\begin{array}{l}\text { Gasolin } \\
\mathrm{a}\end{array}$ & E5 & E10 & E15 & GD5 & GD10 & GD15 \\
\hline $\mathrm{CO}(\%)$ & 3 & 5.95 & 1.26 & 0.99 & 0.14 & 3.26 & 3.51 & 2.4 \\
\hline $\mathrm{CO} 2(\%)$ & $\begin{array}{c}13- \\
16.5\end{array}$ & 13.55 & 9.86 & 6.49 & 5 & 7.46 & 8.21 & 7.9 \\
\hline $\begin{array}{l}\mathrm{HC} \\
(\mathrm{ppm})\end{array}$ & 400 & 448 & 377 & 393 & 282 & 307 & 299 & 791 \\
\hline $\begin{array}{l}\text { NOx } \\
(\mathrm{ppm})\end{array}$ & N/A & 11 & 5 & 11 & 6 & 8 & 10 & 17 \\
\hline
\end{tabular}

Por otro lado, se agregó hidrógeno en forma de gas en la toma de aire del carburador, el motor se aceleró, lo que mostró condiciones de velocidad oscilantes y con esto una operación completamente inestable hasta que se desconectó la alimentación de hidrógeno a la mezcla carburante.

Además, se mezcló gasolina con biodiesel al 5\% y porcentajes mayores, el motor tendía a detenerse, se modificó manualmente la apertura de la mariposa del carburador, para que pasara mayor cantidad de mezcla, con lo que aceleraba y desaceleraba de manera muy inestable, los gases $\mathrm{HC}$ se incrementaron demasiado y tampoco se logró estabilizar el motor para las buenas mediciones. 


\section{Conclusiones}

De los resultados obtenidos con las mezclas se obtiene el mejor rendimiento con la mezcla de gasolina con diésel al 5\%, con $18 \mathrm{seg} / \mathrm{ml}$, sin embargo, el valor de CO es $3.26 \%$ mayor al límite de $3 \%$, lo cual indica que no se quema todo el carbono y por lo mismo se considera que no llega al límite del $\mathrm{CO} 2$, para la misma mezcla se tiene un valor de $\mathrm{HC}$ menor al límite, lo que indica un buen desempeño en cuanto a emisiones y rendimiento, esta mezcla mantiene muy estable el motor en velocidad y el incremento de temperatura de operación, con respecto al funcionamiento solo con gasolina, no es notorio para el valor de velocidad elegido. Por otro lado, el siguiente valor cercano en rendimiento es la mezcla de gasolina con etanol al $10 \%$, con $16 \mathrm{seg} / \mathrm{ml}$, con valores de lectura menores en $\mathrm{CO}$ y $\mathrm{CO} 2$, lo cual, se esperaba debido a que se reduce el carbono al agregar etanol, para este experimento se presentó el mayor incremento de temperatura con un valor cercano a $89^{\circ} \mathrm{C}$, con respecto al funcionamiento solo con gasolina que es cercano a $86^{\circ} \mathrm{C}$.

Se experimentó también con porcentajes de etanol del 20\% mezclados con gasolina, sin embargo, el ducto (manguera de hule) presentó fugas ligeras por lo que se detuvo la prueba. Se reemplazó el ducto con otro nuevo y se realizó el experimento con mezcla de gasolina etanol al $50 \%$, pero también se encontraron fugas de la mezcla y se concluyó que el etanol en altos porcentajes daña el ducto de este tipo. Cuando se mezcla gasolina con diésel en porcentajes mayores al $20 \%$, el motor no logra arrancar, pero si se arranca con gasolina y después se agrega la mezcla, si mantiene la regulación de velocidad, la temperatura aumenta ligeramente, pero el rendimiento no se incrementa. De [21] se obtiene el precio promedio al $19 / 10 / 2021$ por 1 litro, gasolina premium $=\$ 21.60 \mathrm{mx}$, diésel $=\$ 21.82 \mathrm{mx}$.

Para el régimen de velocidad gobernada, en el que se propone funciona el motor: Si se utiliza 95\% de gasolina y $5 \%$ de diésel, se tiene un rendimiento de 1.5 veces con respecto al rendimiento de solo usar gasolina, es decir, si se usa la mezcla durante $5 \mathrm{~h}=18,000 \mathrm{~s}$, se gastaría 1 litro de mezcla, que costaría $21.60 * 0.95+21.82 * 0.05=\$ 21.61 \mathrm{mx}$.

Al comparar con el uso solo de gasolina, funcionando durante $5 \mathrm{~h}=18,000 \mathrm{~s}$, se gastaría 1.5 litros, lo que constaría $21.60 * 1.5=\$ 32.40 \mathrm{mx}$. Lo que implica ahorro económico del $33.33 \%=(1-$ $(21.6 / 32.4))^{*} 100 \%$, para el precio actual de los combustibles.

Como trabajo a futuro queda pendiente la modificación del carburador para ingresar combustibles gaseosos y mantener la regulación de velocidad para analizar el funcionamiento. Además de un análisis económico al utilizar la mezcla de combustibles por ejemplo en una planta de generación eléctrica con velocidad gobernada.

Se agradece el préstamo del equipo analizador de gases de escape para el MCI (AGA5000 VEI) al personal del CONALEP plantel Chetumal Lic. Jesús Martínez Ross, para la obtención de datos por las emisiones.

\section{Referencias}

[1] Ecomotion. ecomotion.es/articulos-online/los-motores-de-combustion-interna-representantodavia-mas-del-90-de-las-ventas-mundia-nz6Uh (accesado el 17 de agosto de 2021).

[2]. El Financiero. https://www.elfinanciero.com.mx/cdmx/2021/04/27/contingencia-ambiental-semantiene-en-el-valle-de-mexico/ (accesado el 18 de agosto de 2021)

[3]. Secretaría del Medio Ambiente. https://sedema.cdmx.gob.mx/programas/programa/hoy-nocircula (accesado el 18 de agosto de 2021).

[4]. Salinas-Callejas, E. y Gasca-Quezada, V. Los biocombustibles. El cotidiano, 2009, 157, 75-82.

[5]. Guo, M.; Song, W.; Buhain, B. (2014). Bioenergy and biofuels: History, status, and perspective. Sustainable Energy Reviews, 42(1), 712-725. 
[6]. Yang, Y.; Zhilin, T.; Yuanfeng, L.; Shu, W.; Hao, C. An overview of biofuel power generation on policies and finance environment, applied biofuels, device and performance. J. of Traffic and Transportation Engineering. 2021.

[7]. Secretaría de Energía (SENER). Prospectiva de Energías Renovables 2016-2030. 2016, México.

[8]. Nguyen-Duca, K.; Nguyen-Tien, H.; Nguyen-Duy, V. Performance enhancement and emission reduction of used motorcycles using flexible fuel technology. Energy Institute. 2018, 91, 145-152.

[9]. Perez-Sanchez, A.; Montero-Alpirez, G.; Ayala-Bautista, R.; Coronado-Ortega, M.; CampbellRamírez, H.;

García-González, C. Aspen simulation of diesel biodiesel blends combustion. Ingeniería, Investigación y Tecnología. 2015, 16, 83-92.

[10]. Oliveros T., C.E.; Sanz U., J.R.; Rodríguez V., N. Evaluación de un gasificador de flujo descendente utilizando astillas de madera de café. Revista Cenicafé. 2017, 68, 61-75.

[11]. Badillo-Hernández, U.; Santamaría-Padilla, L.A.; Álvarez, J.; Álvarez-Icaza, L. Input-state estimation of a spatially distributed tubular gasification reactor. IFAC-PapersOnLine. 2019, 52, 212-217.

[12]. Suozhu, P.; Kai, C.; Min, C.; et all. Experimental study on the cyclic variations of ethanol/diesel reactivity controlled compression ignition (RCCI) combustion in a heavy-duty diesel engine. Energy. 2021, 237, 121614.

[13]. Vergel-Ortega, M; Valencia-Ochoa, G.; Duarte-Forero, J. Experimental study of emissions in single cylinder diesel engine operating with diesel-biodiesel blends of palm oil-sunflower oil and ethanol. Case Studies in Thermal Engineering. 2021, 26.

[14]. Souza, J.; Dambros, G.; Altafini, C.; Wander, P.; Oliveira, L. Dual fuel ethanol port injection in a compression ignition diesel engine: Technical analysis, environmental behavior, and economic viability. Journal of Cleaner Production. 2021, 308, 127396.

[15]. Chapman, S. J. Maquinas Electricas, 5ta ed.; New York: Mc Graw Hill. 2012. pp. 150-204.

[16]. Castillo-Hernández, P.; Mendoza-Domínguez, A.; Caballero-Mata, P. Analysis of Physicochemical Properties of Mexican Gasoline and Diesel Reformulated with Ethanol. Ingeniería Investigación y Tecnología. 2012, 8, pp 293-306.

[17]. Becerra, G.; Osorio, E.; Sanchez, V.M.; Aguilar, J.O.; et all. Sizing of electrolyzer for application in combustion engines. Procedings of the XVII International Congress of the Mexican Hydrogen Society, Guanajuato, 2017, pp. 1-10.

[18]. Ford. https://www.ford.mx/blog/experto/diferencias-tipos-gasolina-correcto-vehiculo$\underline{201902 /}$

(accesado el 18 de octubre de 2021).

[19]. Torres González, M. Determinación del índice de cetano aproximado, la viscosidad cinemática y las propiedades reológicas del biodiesel a nivel laboratorio. Tesis de Ing.

Ambiental, UDLAP 2020.

[20]. Priya; Singh Deora, P.; et all. Biofuels: An alternative to conventional fuel and energy source. Materials Today: Proceedings. 2021. doi.org/10.1016/j.matpr.2021.08.227.

[20]. Becerra, G.; Chi, N.; Avilés, J.D.; Aguilar, J.O.; et all. Control de cuerpo de aceleración para pruebas con mezclas de combustible en MCI. Memorias del Congreso Nacional de Control Automático. 2019. pp 535-539.

[21]. Gasolina México. https://gasolinamexico.com.mx/estados/quintana-roo/othon-p-blanco/ (accesado el 19 de octubre de 2021). 